\title{
On the magnetism, thermal- and electrical transport of $\mathrm{SrMoO}_{2} \mathrm{~N}$
}

\author{
D. Logvinovich, ${ }^{1}$ J. Hejtmánek ${ }^{2}$ K. Knižek ${ }^{2}$ M. Maryško, ${ }^{2}$ N. Homazava, ${ }^{3}$ P. Tomeš,${ }^{2}$ \\ R. Aguiar, ${ }^{4}$ S. G. Ebbinghaus, ${ }^{5}$ A. Reller, ${ }^{4}$ and A. Weidenkaff ${ }^{1, a)}$ \\ ${ }^{1}$ Solid State Chemistry and Catalysis, EMPA, Ueberlandstrasse 129, CH-8600 Duebendorf, Switzerland \\ ${ }^{2}$ Institute of Physics, Academy Sciences of the Czech Republic, Cukrovarnická 10, CZ-15263, Prague 6, Czech \\ Republic \\ ${ }^{3}$ Analytical Chemistry, EMPA, Ueberlandstrasse 129, CH-8600 Duebendorf, Switzerland \\ ${ }_{5}^{4}$ Solid State Chemistry, University of Augsburg, D-80159 Augsburg, Germany \\ ${ }^{5}$ Solid State Chemistry, Martin-Luther-Universität Halle-Wittenberg, Kurt-Mothers Strasse 2, D-06120 Halle \\ (Saale), Germany
}

(Received 25 July 2008; accepted 4 December 2008; published online 29 January 2009)

Physical properties of perovskite-type $\mathrm{SrMoO}_{2} \mathrm{~N}$ phases were studied in the temperature range of $3 \mathrm{~K}<T<300 \mathrm{~K}$. The oxynitride crystallizes in a cubic unit cell (space group $\operatorname{Pm} \overline{3} m$ ) as revealed by neutron and $\mathrm{x}$-ray diffraction measurements. The polycrystalline material shows weakly temperature dependent electrical resistivity and low glasslike heat conductivity, both reflecting the unusual strength of the scattering processes in the charge carrier transport. Based on the positive Seebeck coefficient values, holes are identified as the dominating charge carriers in $\mathrm{SrMoO}_{2} \mathrm{~N}$. Down to $150 \mathrm{~K}$, the magnetic susceptibility is temperature independent and explained as enhanced Pauli paramagnetism $\left(\chi \sim 10^{-4} \mathrm{emu} \mathrm{mol}^{-1} \mathrm{Oe}^{-1}\right)$. The absolute value of its magnetic susceptibility is, however, half of that for $\mathrm{SrMoO}_{3}$. Simultaneously, the lower Sommerfeld coefficient $\gamma$ measured for the oxynitride confirms the lower density of states near the Fermi level for $\mathrm{SrMoO}_{2} \mathrm{~N}$ compared to $\mathrm{SrMoO}_{3}$. At low temperature, both $\mathrm{SrMoO}_{2} \mathrm{~N}$ and $\mathrm{SrMoO}_{3}$ show Curie paramagnetism superimposed to the temperature independent Pauli paramagnetism and an anomaly at $T=54 \mathrm{~K}$. This anomaly is attributed to the presence of molecular oxygen in the material, while the Curie upturn is likely associated with a small amount of paramagnetic centers. () 2009 American Institute of Physics. [DOI: 10.1063/1.3067755]

\section{INTRODUCTION}

Partial substitution of oxygen by nitrogen offers an alternative to cationic substitution for altering physical properties of perovskites, $\mathrm{ABO}_{3}$. Thus, substitution of oxygen by nitrogen in perovskite-type oxides with $d^{0}$ configuration of the transition metal leads to an optical band gap reduction. The actual band gap value can be effectively tuned by varying the nitrogen content with cross substitution. ${ }^{1}$ This makes perovskite-type oxynitrides (also be named nitride oxides) with the general formula $A B \mathrm{O}_{3-x} \mathrm{~N}_{x}, A=\mathrm{AE}, \mathrm{RE}$, and $B$ $=\mathrm{Ti}^{4+}, \mathrm{Ta}^{5+}, \mathrm{Nb}^{5+}$ prospective for optical ${ }^{2,3}$ and photocatalytic $^{4,5}$ applications.

Since $\mathrm{N}^{3-}$ ions have a lower oxidation state than $\mathrm{O}^{2-}$ ions, partial substitution of oxygen with nitrogen in conducting oxide perovskites (with $d^{n}$ configuration of the transition element) can be used to change the charge carrier concentration and consequently the electronic and magnetic properties of these materials. ${ }^{3,6,7}$

Cubic perovskite $\mathrm{SrMoO}_{3}\left(d^{2}\right)$ exhibits high charge carrier density and optical transparency in the ultraviolet region. ${ }^{8,9}$ Therefore, this transparent oxide is a potential candidate for diverse applications, including solar cells. ${ }^{8,9}$ Partial substitution of oxygen in $\mathrm{SrMoO}_{3}$ with nitrogen allows to generate mixed oxidation states of molybdenum and to explore new electronic and magnetic properties of the compounds.

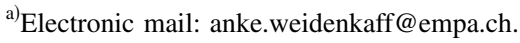

Mixed-valent molybdenum oxynitride perovskites are a rather poorly investigated group of materials. Until now, $\mathrm{SrMoO}_{2.6} \mathrm{~N}_{0.4}$ and $\mathrm{SrMoO}_{2.5} \mathrm{~N}_{0.5}(x=0.4,0.5)$ are the only reported powder and thin-film compositions. ${ }^{10-12}$ The magnetic measurements reveal Pauli paramagnetism $(\chi$ $\left.\sim 10^{-4} \mathrm{emu} \mathrm{mol}^{-1} \mathrm{Oe}^{-1}\right)$ at room temperature and a magnetic transition at $T \sim 54 \mathrm{~K}$. This anomaly is referred to an antiferromagnetic transition ${ }^{10}$ or to a spin-glass formation. ${ }^{11}$

Recently, we succeeded in preparing $\mathrm{CaMoO}_{1.7} \mathrm{~N}_{1.3}$, $\mathrm{BaMo}(\mathrm{O}, \mathrm{N})_{3},{ }^{13}$ and $\mathrm{SrMoO}_{3-x} \mathrm{~N}_{\mathrm{x}}(x>1){ }^{14}$ compounds. Electronic properties of $\mathrm{SrMoO}_{3-x} \mathrm{~N}_{x}(x>1)$ have been measured at temperatures of $340 \mathrm{~K}<T<950 \mathrm{~K}^{14}$ These novel materials showed low electrical conductivity $\left(25-80 \mathrm{~S} \mathrm{~cm}^{-1}\right)$ and a positive Seebeck coefficient $\left(15-30 \mu \mathrm{V} \mathrm{K}^{-1}\right)$, a value being about one order of magnitude higher than the reported one for $\mathrm{SrMoO}_{3}{ }^{15}$ To understand the influence of anionic substitution on the charge carriers subsystem, we compare in this work low temperature physical properties of the Mo oxynitride with those of the similarly prepared $\mathrm{SrMoO}_{3}$.

\section{EXPERIMENTAL}

A quantity of $0.04 \mathrm{~mol} \mathrm{MoO}_{3}$ (JMC, Specpure) was dissolved in a minimal amount of $\mathrm{NH}_{3}$ (aqueous, 25\%) and precipitated with $100 \mathrm{ml}$ of an aqueous solution of $0.4 M$. $\mathrm{Sr}\left(\mathrm{NO}_{3}\right)_{2}$ (Merck, $\left.>99 \%\right)$. The precipitate was washed with 


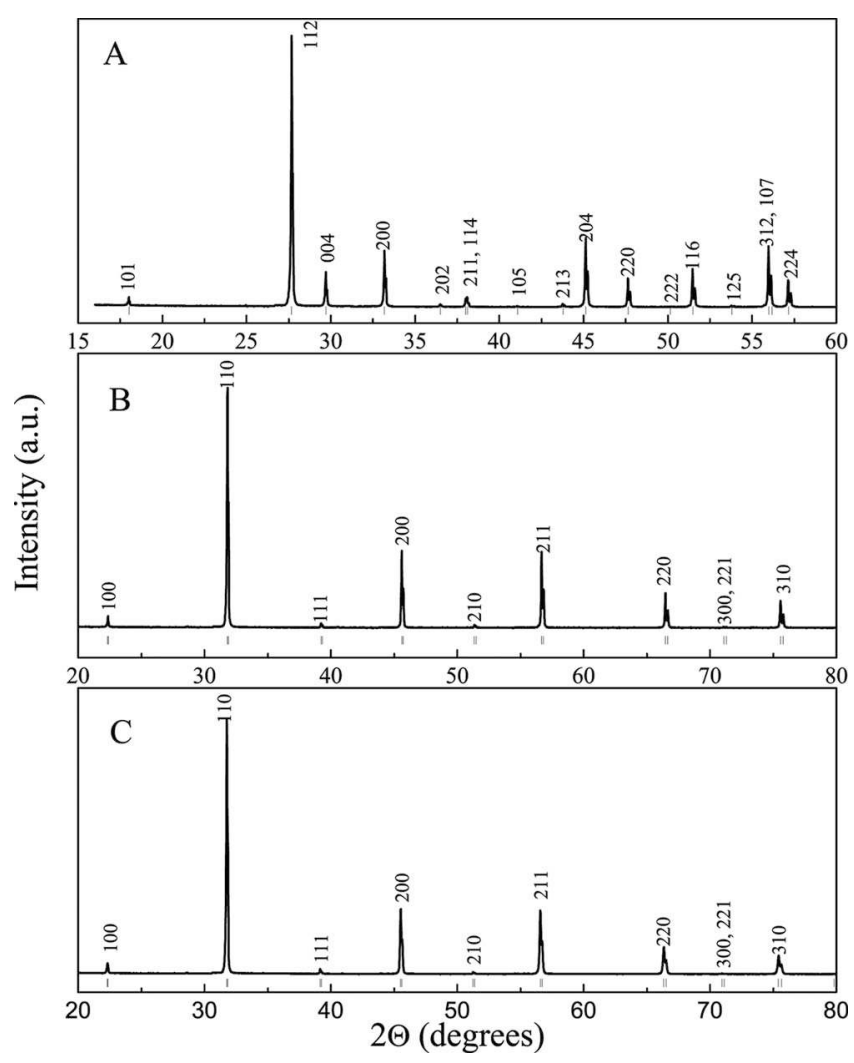

FIG. 1. XRD patterns confirming the phase purity of (a) $\mathrm{SrMoO}_{4}$ after annealing at $1073 \mathrm{~K}$ for $4 \mathrm{~h}$. Space group: $14_{1} / a$. The reflections are assigned according to the pdf entry 85-0809; (b) $\mathrm{SrMoO}_{3}(P m \overline{3} m$, pdf entry 81-0640) obtained by the reduction of $\mathrm{SrMoO}_{4}$; (c) $\mathrm{SrMoO}_{2} \mathrm{~N}(P m \overline{3} m)$ obtained by thermal ammonolysis of $\mathrm{SrMoO}_{4}$.

distilled water, dried, and annealed at $1073 \mathrm{~K}$ for $4 \mathrm{~h}$ to form phase pure well crystalline $\mathrm{SrMoO}_{4}$ powder as confirmed by X-ray diffraction (XRD) [Fig. 1(a)].

$\mathrm{SrMoO}_{3}$ was prepared by reduction of $1 \mathrm{~g}$ of freshly synthesized $\mathrm{SrMoO}_{4}$ at $T=1373 \mathrm{~K}$ with forming gas $\left(5 \% \mathrm{H}_{2} / 95 \% \mathrm{~N}_{2}, 99.999 \%\right.$ purity, Pangas). Under the gas flow of $300 \mathrm{ml} / \mathrm{min}$, the reduction was completed in $5 \mathrm{~h}$.

The thermal ammonolysis of $\mathrm{SrMoO}_{4}$ was performed in a rotating cavity reactor described elsewhere. ${ }^{1}$ A quantity of $2.25 \mathrm{~g}$ of the $\mathrm{SrMoO}_{4}$ powder was ammonolyzed at $T$ $=1073 \mathrm{~K}$ for $11 \mathrm{~h}$ under ammonia (PanGas, $>99.98 \%)$ with a gas flow of $150 \mathrm{ml} / \mathrm{min}$. The synthesis temperature was reached with a heating rate of $10 \mathrm{~K} / \mathrm{min}$. After the reaction, the furnace was cooled down to room temperature with a cooling rate of $10 \mathrm{~K} / \mathrm{min}$ under flowing ammonia.

The electrical transport measurements were performed on bars with dimensions of $1.65 \mathrm{~mm} \times 5-10 \mathrm{~mm} \times 1 \mathrm{~mm}$. The bars were obtained by applying a uniaxial pressure of 10 bar followed by a cold isostatic pressing at 2000 bar. The bars of the oxynitride material were sintered at $T=1073 \mathrm{~K}$ for $30 \mathrm{~min}$ under flowing ammonia. The bars of $\mathrm{SrMoO}_{3}$ were sintered at $T=1373 \mathrm{~K}$ for $4 \mathrm{~h}$ under flowing forming gas. The measured density of the obtained ceramics was about $75 \%$ of the theoretical density.

Structure and phase purity of the obtained oxynitrides were studied by XRD using a Phillips X'Pert PRO MPD $\Theta-\Theta$ system equipped with an $X^{\prime}$ Celerator linear detector. The $\mathrm{O} / \mathrm{N}$ content of the different samples was measured by the hotgas-extraction method using a LECO TC500 analyzer. Silicon nitride and silicon oxide were used as calibration standards for nitrogen and oxygen, respectively.

The neutron diffraction (ND) data were recorded at the high resolution powder diffractometer for thermal neutrons (HRPT) (Ref. 16) located at the Swiss Spallation Neutron Source (SINQ) of the Paul Scherrer Institut in Switzerland. The measurements were performed with a neutron wavelength $\lambda=1.494 \AA$ in the angular range of $10^{\circ}-150^{\circ}$ with a step size of $0.05^{\circ}$. The background was fitted with a sixcoefficients polynomial function. The peak shape was modeled using a Tompson-Cox-Hastings pseudo-Voigt profile function. Structure refinements were performed using FULLPROF. $^{17}$

The microstructure of the samples has been studied with scanning electron microscopy (SEM) using a LEO JSM$6300 \mathrm{~F}$ electron microscope. The cationic compositions study was made by energy dispersive X-ray spectrometry (EDXSLink Pentafet 5947, from Oxford Microanalysis group).

To check for possible magnetic impurities ( $\mathrm{Fe}, \mathrm{Co}, \mathrm{Ni}$ ) chemical analysis by inductively coupled plasma mass spectrometry was carried out. The measurements were performed with inductively coupled plasma quadrupole measurement system (ICP-QMS) ELAN 6000 instrument (Perkin Elmer/ Sciex, Canada).

Low temperature physical properties measurements of $\mathrm{SrMoO}_{3}$ and $\mathrm{SrMoO}_{2} \mathrm{~N}$ (see below) were performed in the temperature range $T=5-300 \mathrm{~K}$ using a Quantum Design physical property measurement system (PPMS). Magnetic susceptibility measurements were done by using a vibrating sample magnetometer (VSM) (Quantum Design PPMS) and a SQUID (superconducting quantum interference device) (Quantum Design magnetic poperty measurement system). The obtained values were corrected for diamagnetic contributions of the sample holder and the low temperature varnish, ionic core diamagnetism, and Van-Vleck paramagnetism. ${ }^{18}$ Field dependent isothermal magnetization measurements were performed at room and low temperatures.

\section{RESULTS AND DISCUSSION}

XRD measurements confirm phase purity of $\mathrm{SrMoO}_{4}$, $\mathrm{SrMoO}_{3}$ and the oxynitride samples obtained after $11 \mathrm{~h}$ of ammonolysis at $T=1073 \mathrm{~K}$ (Fig. 1). The cationic composition study reveals a Sr:Mo ratio of the oxide and oxynitride samples equal to $0.98(1): 1.02(2)$. Thus, within the experimental error $(\sim 3 \%)$, the obtained ratio is identical with the nominal ratio $1: 1$. The lattice parameter, $a=3.9752(1) \AA$, refined from the XRD data of $\mathrm{SrMoO}_{3}$, is in perfect agreement with the previously reported value of $3.9751(3) \AA .{ }^{15}$ The $\mathrm{O} / \mathrm{N}$ content measured for the oxynitride by hotgas extraction corresponds to the composition $\mathrm{SrMoO}_{1.95(5)} \mathrm{N}_{1.05(5)}$, i.e., within a $1 \sigma$-range integer values were found. The sample is therefore denoted as $\mathrm{SrMoO}_{2} \mathrm{~N}$ in the following. During the reaction of $\mathrm{SrMoO}_{4}$ with ammonia, a significant microstructural reorganization occurs in the particles since the starting oxide and the oxynitride reveal different crystallographic structures and anionic compositions. Thus, the resulting ox- 


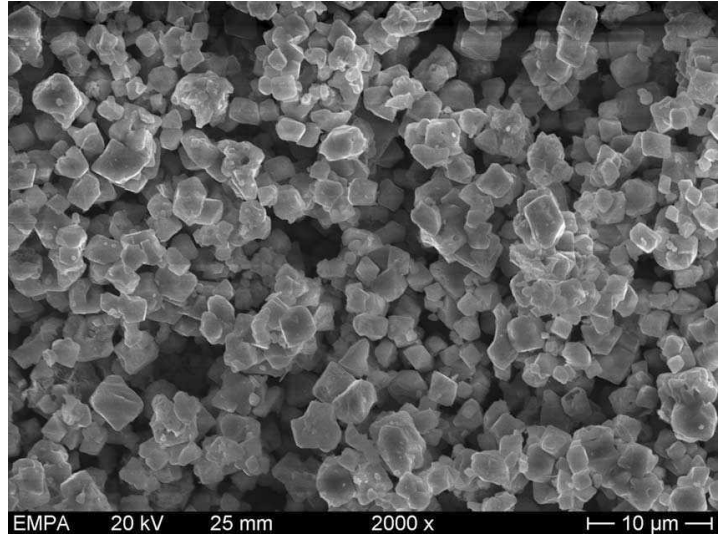

FIG. 2. Microstructure of $\mathrm{SrMoO}_{2} \mathrm{~N}$.

ynitride phases reveal a different particle size (larger surface area) compared to the starting compound. The physical properties of powder materials depend on the particle size, shape, and surface area, which have to be monitored carefully and taken into account for the interpretation of the measured data. Therefore, before performing physical and chemical properties measurements, the particle size of $\mathrm{SrMoO}_{2} \mathrm{~N}$ was determined. SEM investigations on $\mathrm{SrMoO}_{2} \mathrm{~N}$ reveal particles with an average diameter of $\sim 1 \mu \mathrm{m}$ (Fig. 2), which are suitable for the bulk physical properties measurements.

Rietveld refinement of the $\mathrm{SrMoO}_{2} \mathrm{~N}$ powder neutron data was carried out in the space group $P m \overline{3} m$. In the starting structural model, the Sr:Mo ratio was fixed to 1:1 (based on the cationic compositions study), and the $\mathrm{O}: \mathrm{N}$ ratio was set to $2: 1$. Thermal displacement factors were refined isotropically for all atoms. In the next refinement steps, the occupancy factors for oxygen and nitrogen were refined with the anionic site constrained to be fully occupied. In succeeding runs, the occupancy factors of the anions were refined independently. Finally, the lattice and the profile parameters, $2 \Theta_{0}$, the background coefficients, the thermal displacement factors, and the anionic occupancies were refined together. Within the doubled standard deviation $(2 \sigma)$, no difference between the parameters obtained with the constrained and the unconstrained model were found. For this reason, in the final refinement, a complete occupation of the anionic sites was assumed and only the $\mathrm{O} / \mathrm{N}$ ratio was refined. Refinements of the cationic occupancies gave no hints for the presence of the cationic vacancies. The statistics of the refinement and the visual inspection of the fit (Fig. 3) indicate that the model was chosen correctly. The refined $\mathrm{O} / \mathrm{N}$ content corresponds to the composition $\mathrm{SrMoO}_{1.89(2)} \mathrm{N}_{1.11(2)}$, which is in a reasonable agreement with the result obtained by hotgas extraction.

Figure 4(a) shows the temperature dependence of resistivity measured for $\mathrm{SrMoO}_{3}$ and $\mathrm{SrMoO}_{2} \mathrm{~N}$. The resistivity of $\mathrm{SrMoO}_{3}$ is significantly higher than the value reported in literature $(\sim 5 \mu \Omega \mathrm{cm})$ measured on the single crystal. ${ }^{19}$ This underlines that the limiting factor in the electrical transport is the grain-boundary scattering processes. However, despite of the approximately two orders higher resistivity, the synthesized polycrystalline $\mathrm{SrMoO}_{3}$ ceramics show a metallic-like behavior of the electrical resistivity. Metallic-

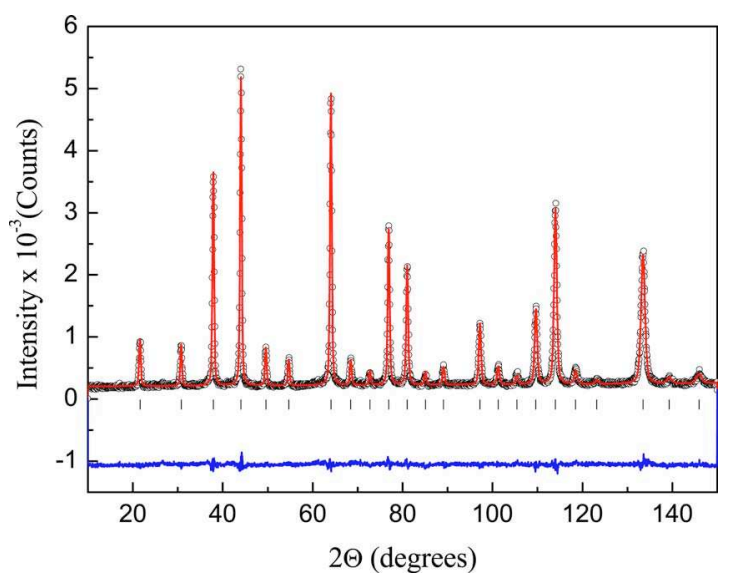

FIG. 3. (Color online) Rietveld refinement plot of the ND data for $\mathrm{SrMoO}_{2} \mathrm{~N}$. Space group: $\operatorname{Pm} \overline{3} m, a=3.9835(1) \AA, \quad w R_{p}=0.0625, R_{p}$ $=0.0469$, and $\chi^{2}=1.56$.

like nature of $\mathrm{SrMoO}_{3}$ is also corroborated by its very low value of the Seebeck coefficient [Fig. 4(b)], which, unlike resistivity, is related to the bulk property of the material. The measured Seebeck values are close to the reported values ${ }^{15,20}$ and the Seebeck coefficient, despite the fact that it is very small, decreases continuously with temperature. This confirms that holelike carriers dominate the charge transport in $\mathrm{SrMoO}_{3}$. Figure 4(c) shows the temperature variation of thermal conductivity. The values of thermal conductivity are about three times lower than those reported in Ref. 20. This effect can be attributed to the lower density of our sample.

Measured magnetic susceptibility data reveal linear paramagnetic response. However, close inspection of magnetization curves at small fields unveiled that initial magnetic susceptibility is significantly higher than that at fields exceeding

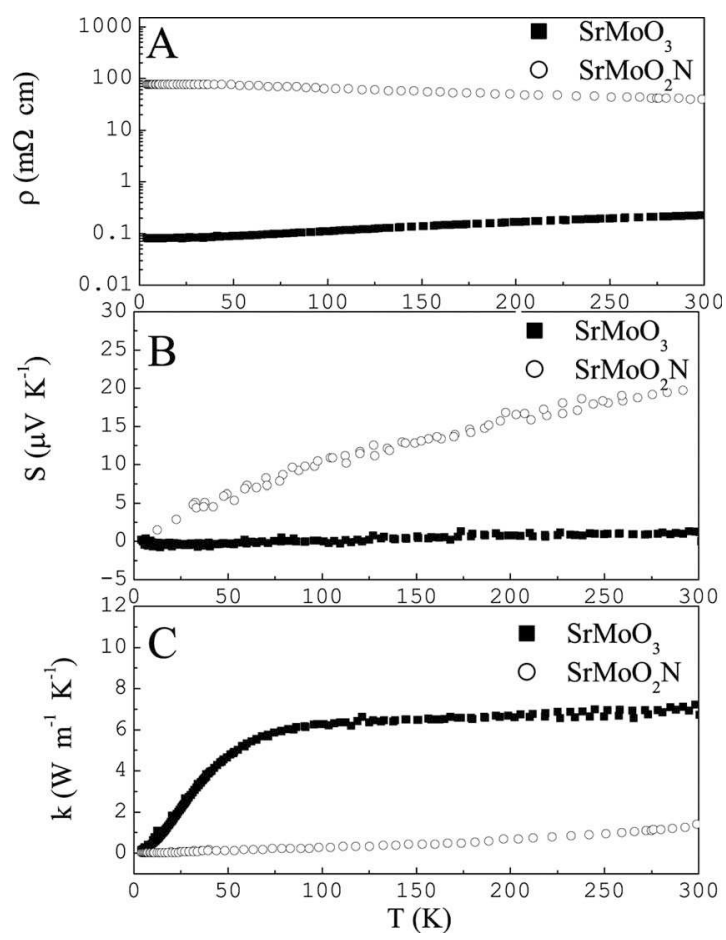

FIG. 4. Temperature dependences of (a) resistivity; (b) Seebeck coefficient, and (c) heat conductivity of $\mathrm{SrMoO}_{3}$ and $\mathrm{SrMoO}_{2} \mathrm{~N}$. 


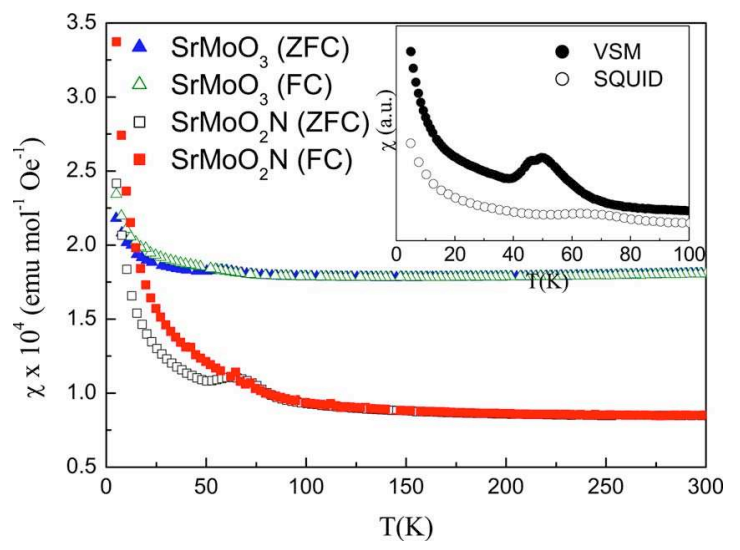

FIG. 5. (Color online) Molar magnetic susceptibility of $\mathrm{SrMoO}_{3}$ and $\mathrm{SrMoO}_{2} \mathrm{~N}$ measured with a SQUID magnetometer. The inset shows an expansion of the magnetic anomaly for $\mathrm{SrMoO}_{3}$ when changing the magnetometer type from SQUID to VSM.

$1 \mathrm{~T}$. This feature was observed independently on temperature and field. Based on the ICP-QMS measurements, we attribute it to the presence of $\mathrm{Ni}$ and Fe traces (5-10 ppm) in our sample. Consequently, to minimize the role of this ferromagnetic impurity on the measured susceptibility, we adopted the conventional scenario, i.e., we applied sufficiently high field to saturate it. Accordingly, the zero field cooled and field cooled susceptibility measurements were performed under an applied field of $2 \mathrm{~T}$.

As shown in Fig. 5, $\mathrm{SrMoO}_{3}$ exhibits a temperature independent paramagnetism $\left(\chi \sim 1.8 \times 10^{-4} \mathrm{emu} \mathrm{mol}^{-1} \mathrm{~K}^{-1}\right)$ between $T=100 \mathrm{~K}$ and $T=300 \mathrm{~K}$. This is consistent with the absolute value of magnetic susceptibility reported previously and explained as enhanced Pauli paramagnetism. ${ }^{11} \mathrm{Be}-$ low $100 \mathrm{~K}$, the magnetic susceptibility slightly increases, according to a Curie behavior superposed to the temperature independent Pauli paramagnetism. Finally at $T \sim 54 \mathrm{~K}$ a monotonous hyperbolic increase is interrupted by a weak anomaly (see below).

Unlike $\mathrm{SrMoO}_{3}$, the oxynitride $\mathrm{SrMoO}_{2} \mathrm{~N}$ shows semiconductinglike behavior of its resistivity with temperature [Fig. 4(a)]. The thermal behavior of the electrical resistivity is reminiscent to that observed for glasses, i.e., systems with disorder on the atomic scale. Moreover, the absolute resistivity of $\mathrm{SrMoO}_{2} \mathrm{~N}$ is higher than that of $\mathrm{SrMoO}_{3}$. Based on the measured absolute Seebeck values, it can be deduced that holes are the predominating mobile charge carriers of $\mathrm{SrMoO}_{2} \mathrm{~N}$ [Fig. 4(b)]. The Seebeck values of $\mathrm{SrMoO}_{2} \mathrm{~N}$ are significantly higher than those of $\mathrm{SrMoO}_{3}$, pointing to the lower carrier concentration in $\mathrm{SrMoO}_{2} \mathrm{~N}$.

The measured thermal conductivity value of $\mathrm{SrMoO}_{2} \mathrm{~N}$ is one order of magnitude smaller than that of $\mathrm{SrMoO}_{3}$. It also resembles the thermal conductivity of glasses, which corroborates with the presented behavior of the electrical resistivity [Fig. 4(c)].

The weak temperature dependence of the electrical resistivity together with the very low thermal conductivity of $\mathrm{SrMoO}_{2} \mathrm{~N}$ suggest that the mean free paths of both phonons and charge carriers are close to the interatomic distance. This can be caused by one or a combination of the following factors: (i) creation of the charge carrier traps (local defects, scattering centers) due to the occupation of the same crystallographic site by atoms of different size, charge, and electronic level positions, (ii) dominating role of the charge carriers scattering on grain boundaries and within grains on microdomain boundaries, and (iii) bond length fluctuations in $\mathrm{SrMoO}_{2} \mathrm{~N}$ as suggested from its previous $\mathrm{x}$-ray absorption near-edge spectroscopy study. ${ }^{14}$

$\mathrm{SrMoO}_{2} \mathrm{~N}$ oxynitride shows an analogous behavior as $\mathrm{SrMoO}_{3}$ shows an analogous behavior with respect to a temperature independent paramagnetism down to $T=150 \mathrm{~K}$, a low temperature upturn, and an anomaly at $T \sim 54 \mathrm{~K}$. The temperature independent component is, however, smaller than that of $\mathrm{SrMoO}_{3}$ and the low temperature increase and anomaly are enhanced. The magnetic data of $\mathrm{SrMoO}_{2} \mathrm{~N}$ can be fitted to the following equation:

$$
\chi=\chi_{P+L}+\frac{C}{T-\Theta},
$$

where $\chi$ is the measured value of magnetic susceptibility (corrected for the sample holder, the varnish, and diamagnetic contributions), $\chi_{P+L}$ is the sum of the Pauli paramagnetic and Landau diamagnetic terms, $C$ is the Curie constant, $T$ is temperature, and $\Theta$ is the Weiss constant. The fit results in $\chi_{P+L}=8.5 \times 10^{-5} \mathrm{emu} \mathrm{mol}^{-1}, C=3.310^{-5} \mathrm{emu} \mathrm{mol}^{-1}$ and $\Theta=-63 \mathrm{~K}$ The $C$ value corresponds to the effective magnetic moment of $\mu_{\mathrm{eff}}=0.05 \mu_{B}$. The negative sign of $\Theta$ points to the antiferromagnetic interaction between the moments.

The temperature independent paramagnetic term $\chi_{P+L}$ can be expressed as

$$
\chi_{P+L}=\mu_{B} N D_{F}\left(1-\frac{1}{3}\left(\frac{m}{m^{*}}\right)^{2}\right),
$$

where $D_{F}$ is density of states (DOS) at the Fermi level, $N$ is the number of itinerant electrons per one mole of unit formula, $m$ is the electron mass and $m^{*}$ is the effective electron mass. Within the free electron approximation $m=m^{*}$, hence,

$$
\chi_{P+L} \approx 0.7 \mu_{B} N D_{F} .
$$

From this equation, the susceptibility related DOS at the Fermi level can be estimated. Its value for $\mathrm{SrMoO}_{2} \mathrm{~N}$ is equal to $D_{F} \sim 25 \mathrm{Ry}^{-1} /$ f.u., while that deduced for $\mathrm{SrMoO}_{3}$ is significantly higher, i.e., $D_{F} \sim 55 \mathrm{Ry}^{-1} /$ f.u.

Alternatively, the DOS at the Fermi level can be calculated from the Sommerfeld coefficient $(\gamma)$, which is derived from the bulk-related heat capacity data. The specific heat data for polycrystalline $\mathrm{SrMoO}_{2} \mathrm{~N}$ are plotted as $C_{P} / T$ versus $T^{2}$ (Fig. 6). Similar as for $\mathrm{SrMoO}_{3},{ }^{19}$ the data follow a straight line below $10 \mathrm{~K}$. The sum of a linear term for the electronic $(\gamma T)$ and the lattice $\left(\beta T^{3}\right)$ contributions is

$$
C_{P}=\gamma T+\beta T^{3},
$$

where

$$
\gamma=\frac{\pi^{2}}{3} K_{B}^{2} N D_{F}
$$




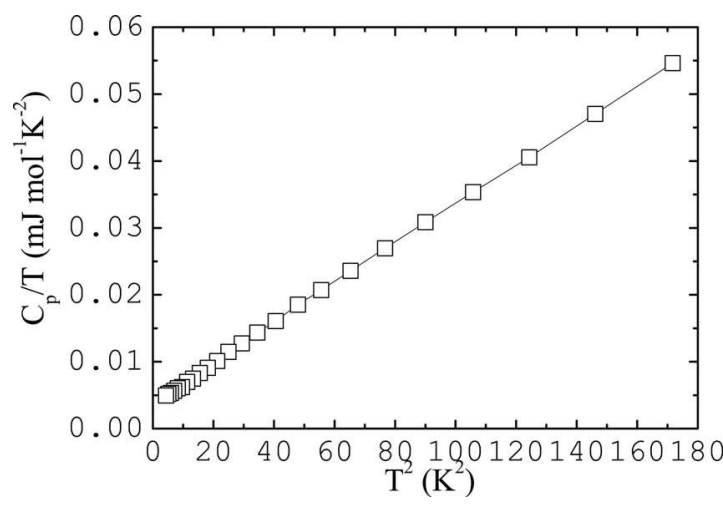

FIG. 6. Temperature dependence of $C_{p} / T(2 \mathrm{~K}<T<13 \mathrm{~K})$ measured on $\mathrm{SrMoO}_{2} \mathrm{~N}$.

$$
\beta=12 \pi^{4} N k_{B} / 5 \Theta_{D}^{3},
$$

where $k_{B}$ is the Boltzmann constant and $\Theta_{D}$ is the Debye temperature.

The fitting to Eq. (4) results in $\gamma=3.9 \mathrm{~mJ} \mathrm{~mol}^{-1} \mathrm{~K}^{2}$ and $\Theta_{D}=320 \mathrm{~K}$. The value of $\gamma$ corresponds to the DOS of $D_{F}$ $=23 \mathrm{Ry}^{-1} /$ f.u., a value very close to that derived from the magnetic susceptibility and evidences the absence of strong electronic correlations in $\mathrm{SrMoO}_{2} \mathrm{~N}$ [(Sommerfeld-Wilson ratio $R_{W} \approx 1$ (Ref. 21)]. Let us note the literature value of $\gamma$ for $\mathrm{SrMoO}_{3}\left(7.9 \mathrm{~mJ} \mathrm{~mol}^{-1} \mathrm{~K}^{2}\right)$, which is higher than that of $\mathrm{SrMoO}_{2} \mathrm{~N}$, and hence the DOS at the Fermi level of $\mathrm{SrMoO}_{3}$ $\left(D_{F} \sim 45.0 \mathrm{Ry}^{-1} /\right.$ f.u. $)$ is higher than that of $\mathrm{SrMoO}_{2} \mathrm{~N}$. To conclude about the influence of the anionic substitution on the magnetic correlations of the $\mathrm{SrMoO}_{3-\mathrm{x}} \mathrm{N}_{\mathrm{x}}$ system, the Sommerfeld-Wilson ratio of $\mathrm{SrMoO}_{2} \mathrm{~N}$ should be compared with that of $\mathrm{SrMoO}_{3}$. From Eq. (3), we obtain the DOS of $55.0 \mathrm{Ry}^{-1}$ / f.u. for $\mathrm{SrMoO}_{3}$ and, the Sommerfeld-Wilson ratio of $\mathrm{SrMoO}_{3}$ is $\sim 1.2$. Based on this analysis, $\mathrm{SrMoO}_{3}$ does not exhibit strong electron correlations.

Lower DOS at $E_{f}$ for $\mathrm{SrMoO}_{2} \mathrm{~N}$ is consistent with an expected increase in the formal Mo oxidation state, e.g., the removal of electrons from the itinerant $t_{2 g}$ states of Mo ions, when part of $\mathrm{O}^{2-}$ in $\mathrm{SrMoO}_{3}$ is replaced with $\mathrm{N}^{3-}$,

$$
\mathrm{SrMo}^{4+} \mathrm{O}_{3}+y \mathrm{NH}_{3}=\mathrm{SrMo}^{(4+y)+} \mathrm{O}_{3-y} \mathrm{~N}_{y}+y \mathrm{H}_{2} \mathrm{O}+y / 2 \mathrm{H}_{2} .
$$

Together with the anionic composition refined from the ND data, this points to the fact that an increase of the molybdenum oxidation state is the main charge compensating mechanism for partial substitution of $\mathrm{O}^{2-}$ with $\mathrm{N}^{3-}$.

The anomaly measured at $T \sim 54 \mathrm{~K}$ apparently cannot be associated with partial substitution of $\mathrm{O}^{2-}$ with $\mathrm{N}^{3-}$ as the same magnetic feature can be noted on the magnetic susceptibility curves of $\mathrm{SrMoO}_{3}$ and $\mathrm{Sr}_{2} \mathrm{MoO}_{4}$ published previously. $^{22,23}$ Although no explanations are given in Ref. 22 , the authors of Ref. 23 have attributed the feature to molecular oxygen. It is known that residual oxygen presence in the sample and/or in the measurement chamber can significantly alter magnetic susceptibility data of weakly magnetic materials. $^{24}$

Bulk oxygen melts at $T=54.4 \mathrm{~K}$ and a paramagnetic $\gamma$-phase transforms into the antiferromagnetic $\beta$-phase at $T$
$=43.8 \mathrm{~K}$. Thus, a comparative magnetic measurement performed with another instrument is used to uncover the presence of molecular oxygen.

Magnetic susceptibility of the same sample was measured under the same experimental conditions additionally with a VSM PPMS option. From Fig. 5 (inset), it is clearly seen that the measurement with VSM gives a sufficiently expanded magnetic signal with two maxima at $T \sim 47$ and 54 $\mathrm{K}$, respectively. We infer that the maximum at $T \sim 54 \mathrm{~K}$ corresponds to the melting point of oxygen and the maximum at $47 \mathrm{~K}$ to the $\beta-\gamma$ transformation of solid oxygen. Furthermore, we have verified that the measurement of an empty sample holder did not reveal any magnetic anomalies between $T=40-60 \mathrm{~K}$. Thus, the measured signals result from the molecular oxygen in the samples and not in the holder. However, it is difficult to conclude where the molecular oxygen is located: on the measured samples surface, in pores between grains or within grains.

Finally, we summarize the three following possible explanations of Curie paramagnetism measured below $50 \mathrm{~K}$ : (i) it is related to intrinsic property of the material and can be concerned with the presence of electronic defects (such as $\mathrm{Mo}_{\mathrm{Mo}}^{\prime}$ ) as it found before for the $\mathrm{Ca}_{1-x} \mathrm{Sr}_{x} \mathrm{VO}_{3}$ system, ${ }^{25}$ (ii) the presence a paramagnetic impurity phase which cannot be detected neither by x-ray nor ND, or (iii) paramagnetism of $\gamma$ oxygen, confined in pores of the measured material.

Thus, lower magnetic susceptibility confirms that the DOS at $E_{f}$ is lowered in $\mathrm{SrMoO}_{2} \mathrm{~N}$ with respect to $\mathrm{SrMoO}_{3}$ while the more pronounced low temperature features indicate (in coherence with difficult synthesis of $\mathrm{SrMoO}_{2} \mathrm{~N}$ ) the higher concentration of localized magnetic moments.

\section{CONCLUSIONS}

Low temperature physical properties of the cubic perovskite polycrystalline phases, $\mathrm{SrMoO}_{2} \mathrm{~N}$ and $\mathrm{SrMoO}_{3}$, have been measured in the temperature range of $5 \mathrm{~K}<T$ $<300 \mathrm{~K}$. These materials are $p$-type conductors as deduced from the Seebeck coefficient measurements. Both compounds show paramagnetism dominated by the temperature independent Pauli term. Smaller Pauli susceptibility and Sommerfeld coefficient values for the oxynitride confirm independently lower DOS near Fermi level compared to that of $\mathrm{SrMoO}_{3}$. This corroborates with the higher valence of Mo in $\mathrm{SrMoO}_{2} \mathrm{~N}$. The similar anionic compositions, refined from the ND data and deduced from hotgas extraction, point to the fact that the increase of the molybdenum oxidation state is the main charge compensating mechanism for partial substitution of $\mathrm{O}^{2-}$ with $\mathrm{N}^{3-}$. Comparison of the DOS values derived from the magnetic susceptibility and heat capacity measurements allows to rule out the presence of strong electronic correlations in $\mathrm{SrMoO}_{2} \mathrm{~N}$.

In contrast to $\mathrm{SrMoO}_{3}$, the oxynitride sample reveals a low thermal conductivity and semiconductinglike electrical resistivity, which point toward short mean free path for phonons and charge carriers and/or low charge carrier mobility in that compound.

Since the electrical resistivity measurements were performed on bars of pressed and sintered powders and not on 
single crystals, the obtained resistivity values are to be corrected concerning the remaining porosity. Nevertheless, the metallic-like behavior of $\mathrm{SrMoO}_{3}$ is verified by comparing our data with data measured on a $\mathrm{SrMoO}_{3}$ monocrystal. ${ }^{19}$ The semiconducting behavior of $\mathrm{SrMoO}_{2} \mathrm{~N}$ is in accordance with its higher Seebeck coefficient, compared to that of $\mathrm{SrMoO}_{3}$ and the possibility of $\mathrm{Mo}(\mathrm{O}, \mathrm{N})_{6}$ octahedra distortion. ${ }^{14}$ Thus, the obtained temperature dependence of the electrical resistivity for $\mathrm{SrMoO}_{3}$ and $\mathrm{SrMoO}_{2} \mathrm{~N}$ seems to be correct. The electrical transport properties, the enhanced Seebeck coefficient, as well as the lower thermal conductivity of $\mathrm{SrMoO}_{2} \mathrm{~N}$ compared to $\mathrm{SrMoO}_{3}$, makes the oxynitride an interesting candidate as potential thermoelectric material.

Complex magnetic measurements and data analysis reveal that molecular oxygen presence in the measured materials is likely responsible for the magnetic anomaly observed at $T \sim 54 \mathrm{~K}$, contrary to previously suggested intrinsic antiferromagnetic or spin-glass transitions. It is also likely that paramagnetic oxygen contributes to the observed increase of the magnetic susceptibility at low temperatures. $\mathrm{SrMoO}_{2} \mathrm{~N}$ monocrystals would be required to verify this statement.

\section{ACKNOWLEDGMENTS}

The authors acknowledge the German Science Foundation (Grant No. DFG-SPP 1136) for the financial support as well as Dr. Denis Sheptyakov (SINQ) for the technical assistance. This work is partly based on the experiments performed at the Swiss Spallation Neutron Source SINQ, Paul Scherrer Institute, Villingen, Switzerland.

${ }^{1}$ D. Logvinovich, A. Borger, M. Dobeli, S. G. Ebbinghaus, A. Reller, and A. Weidenkaff, Prog. Solid State Chem. 35, 281 (2007).

${ }^{2}$ M. Jansen and H. P. Letschert, Nature (London) 404, 980 (2000).

${ }^{3}$ R. Aguiar, D. Logvinovich, A. Weidenkaff, A. Rachel, A. Reller, and S. G. Ebbinghaus, Dyes Pigm. 76, 70 (2008).
${ }^{4}$ A. Kasahara, K. Nukumizu, T. Takata, J. N. Kondo, M. Hara, H. Kobayashi, and K. Domen, J. Phys. Chem. B 107, 791 (2003).

${ }^{5}$ R. Aguiar, Y. Lee, K. Domen, A. Kalytta, D. Logvinovich, A. Weidenkaff, A. Reller, and S. G. Ebbinghaus, Ceramic Materials Research Trends (Nova Science, New York, 2007), pp. 107-133.

${ }^{6}$ P. Antoine, R. Marchand, Y. Laurent, C. Michel, and B. Raveau, Mater. Res. Bull. 23, 953 (1988).

${ }^{7}$ P. Antoine, R. Assabaa, P. L'Haridon, R. Marchand, Y. Laurent, C. Michel, and B. Raveau, Mater. Sci. Eng., B 5, 43 (1989).

${ }^{8}$ H. Mizoguchi, K. Fukumi, N. Kitamura, T. Takeuchi, J. Hayakawa, H. Yamanaka, H. Yanagi, H. Hosono, and H. Kawazoe, J. Appl. Phys. 85, 6502 (1999)

${ }^{9}$ H. Mizoguchi, N. Kitamura, K. Fukumi, T. Mihara, J. Nishii, M. Nakamura, N. Kikuchi, H. Hosono, and H. Kawazoe, J. Appl. Phys. 87, 4617 (2000).

${ }^{10}$ G. Liu, X. Zhao, and H. A. Eick, J. Alloys Compd. 187, 145 (1992).

${ }^{11}$ I. D. Fawcett, K. V. Ramanujachary, and M. Greenblatt, Mater. Res. Bull. 32, 1565 (1997).

${ }^{12}$ I. C. Lekshmi, A. Gayen, and M. S. Hegde, Mater. Res. Bull. 40, 93 (2005).

${ }^{13}$ D. Logvinovich, M. H. Aguirre, J. Hejtmanek, R. Aguiar, S. G. Ebbinghaus, A. Reller, and A. Weidenkaff, J. Solid State Chem. 181, 2243 (2008).

${ }^{14}$ D. Logvinovich, R. Aguiar, R. Robert, M. Trottmann, S. G. Ebbinghaus, A. Reller, and A. Weidenkaff, J. Solid State Chem. 180, 2649 (2007).

${ }^{15}$ L. H. Brixner, J. Inorg. Nucl. Chem. 14, 225 (1960).

${ }^{16}$ P. Fischer, G. Frey, M. Koch, M. Konnecke, V. Pomjakushin, J. Schefer, R. Thut, N. Schlumpf, R. Burge, and U. Greuter, Physica B 276-278, 146 (2000).

${ }^{17}$ J. Rodriguez-Carvajal, Physica B 192, 55 (1993).

${ }^{18} \mathrm{~J}$. H. Van Vleck, Theory of Electric and Magnetic Susceptibilities (Clarendon, Oxford, 1932).

${ }^{19}$ I. Nagai, N. Shirakawa, S. Ikeda, R. Iwasaki, H. Nishimura, and M. Kosaka, Appl. Phys. Lett. 87, 024105 (2005).

${ }^{20}$ T. Maekawa, K. Kurosaki, H. Muta, M. Uno, and S. Yamanaka, J. Alloys Compd. 390, 314 (2005).

${ }^{21}$ K. G. Wilson, Rev. Mod. Phys. 47, 773 (1975).

${ }^{22}$ S. I. Ikeda and N. Shirakawa, Physica C 341-348, 785 (2000).

${ }^{23}$ N. Shirakawa and S. I. Ikeda, Physica C 364-365, 309 (2001).

${ }^{24}$ F. Beuneu, P. Vajda, and O. J. Zogal, Phys. Rev. Lett. 83, 761 (1999).

${ }^{25}$ I. H. Inoue, O. Goto, H. Makino, N. E. Hussey, and M. Ishikawa, Phys. Rev. B 58, 4372 (1998) 